\title{
Begeisterung ist ansteckend
}

In Österreich würden etwa 300 Rheumatologinnen und Rheumatologen benötigt, die erwachsene Patientinnen und Patienten betreuen. Tatsächlich gibt es nur etwas mehr als 200, und nicht alle von ihnen können sich ausschließlich der Rheumatologie widmen. Um das zu ändern und mehr Studentinnen und Studenten auf die Rheumatologie aufmerksam zu machen, riefen PD Dr. Rudolf Puchner (Internist und Rheumatologe in Wels), Prim. Prof. Dr. Christian Dejaco (Landesweiter Dienst für Rheumatologie, Südtiroler Sanitätsbetrieb) und Prim. Dr. Judith Sautner (Präsidentin der ÖGR [Österreichische Gesellschaft für Rheumatologie und Rehabilitation] und Leiterin der 2. Medizinischen Abteilung am LK Korneuburg-Stockerau) 2017 eine Summer School ins Leben.

Im Rahmen der Summer School der Österreichischen Gesellschaft für Rheumatologie und Rehabilitation (ÖGR) sollen Studenten einen neuen Blick auf die Rheumatologie bekommen. Eingebettet ist die Summer School in ein dreistufiges Karriere-Track-Programm der ÖGR. Heuer fand in Saalfelden bereits die 5 . Summer School statt. Die wichtigste Neuerung in diesem Jahr ist die Unterstützung durch die EULAR (European Alliance of Associations for Rheumatology), die erstmals auch vier international renom-
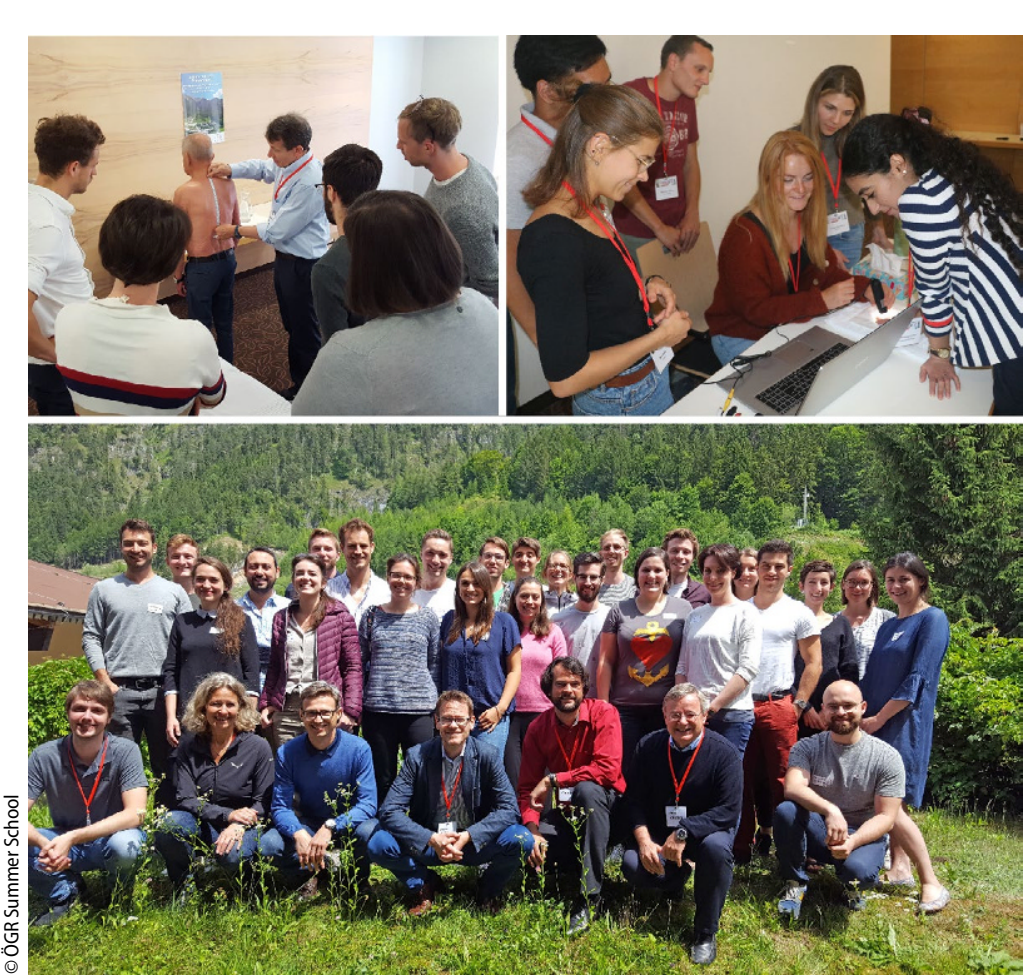

$\Delta$ Eindrücke von der Rheuma Summer School School entsandte. stalten? denten entsteht oft der Eindruck, mierte Referenten zur Summer

Wie kam es dazu, eine Summer School für Rheumatologie zu veran-

Dejaco: Die Summer School wurde von Dr. Puchner, Dr. Sautner und mir initiiert. In Gesprächen mit Studass diese angehenden Ärzte einen völlig falschen Eindruck von der Rheumatologie haben. Das vorherrschende Klischee ist noch immer, dass rheumatische Erkrankungen

School nicht nur Studenten erreichen, die ohnehin bereits an de Rheumatologie interessiert sind, sondern allgemein Studenten auf die Rheumatologie aufmerksam machen und anregen sich intensiver mit der Rheumatologie zu beschäftigen als es das Curriculum der Universitäten vorsieht.

Puchner: Die erste Summer School fand im Juli 2017 statt und traf sofort auf regen Zuspruch. Etwa acht bis zwölf Vortragende unterrichten seither einmal im Jahr etwa 35 Studenten. Der Andrang ist groß, 
Hier steht eine Anzeige.

Springer 
die Teilnehmerzahl ist aber bewusst limitiert, denn neben Vorträgen gibt es auch Workshops, die in Kleingruppen abgehalten werden. Hier werden Fälle diskutiert und besondere Fertigkeiten - wie etwa die klinische Untersuchung, die Ultraschall-Untersuchung und Kapillarmikroskopie - trainiert.

Eine weitere Besonderheit der Summer School ist, dass die Studenten tatsächlich jeweils etwa sechs bis acht Rheuma-Patienten, die die Summer School freiwillig unterstützen, befragen und untersuchen können. Nur heuer konnten, aufgrund der Situation mit COVID-19, keine Patienten anwesend sein.

Sautner: Wir freuen uns auch, wenn die Teilnehmer der ÖGR Summer School in anderen Fächern ihren Weg machen. Einige der Absolventen der Summer School sind in die Rheumatologie gegangen, einige beteiligen sich bereits an rheumatologischen Forschungsprojekten, aber nicht alle. Einige Teilnehmer der ÖGR Summer School sind etwa in der Gastroenterologie angekommen und tragen ihre positiven Erfahrungen mit der Rheumatologie dorthin. Das ist die Basis für gelingende Netzwerke.

Puchner: Unabhängig davon, ob die Studenten letztendlich in der Rheumatologie bleiben: Sie haben im Rahmen der Summer School jedenfalls ein bisschen an der

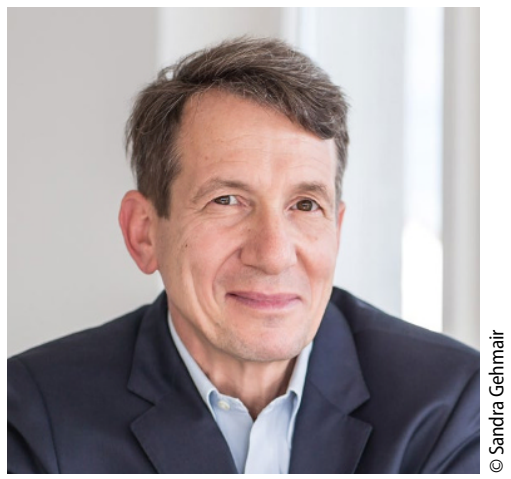

A PD Dr. Rudolf Puchner überschaubaren, 200 Ärzte umfassenden "Rheuma-Familie" angedockt. Ihr neues Verständnis für das Fach Rheumatologie und ihren aufmerksameren Blick für Patienten mit rheumatischen Erkrankungen nehmen sie auf jeden Fall mit.

Inzwischen unterstützt auch die EULAR das Projekt?

Dejaco: In der EULAR gibt es zahlreiche Subkomitees, die unterschiedliche Aufgaben erfüllen. Eines dieser Subkomitees beschäftigt sich mit der Ausbildung, von den Studierenden bis zur Weiterbildung für Fachärzte. Als Teil dieses Komitees hat die ÖGR die österreichische Summer School als Pilotprojekt bei der EULAR beworben.

2021 wurde die ÖGR Summer School dann erstmals auch von der EULAR unterstützt. Das bedeutet unter anderem, dass die EULAR europäische Fachexperten als Vortragende und Tutoren für Workshops zur Unterstützung unseres Teams entsandt hat. Heuer waren das vier Referenten - einer aus Frankreich, einer aus Deutschland und zwei Kolleginnen aus Italien. Diese Rheumatologen hört man für gewöhnlich nur in großen Auditorien sprechen, einen Workshop bei innen besuchen zu können ist also durchaus etwas Besonderes. Von europäischer Seite wird die ÖGR Summer School inzwischen als Pilotprojekt betrachtet, das in Zukunft hof-

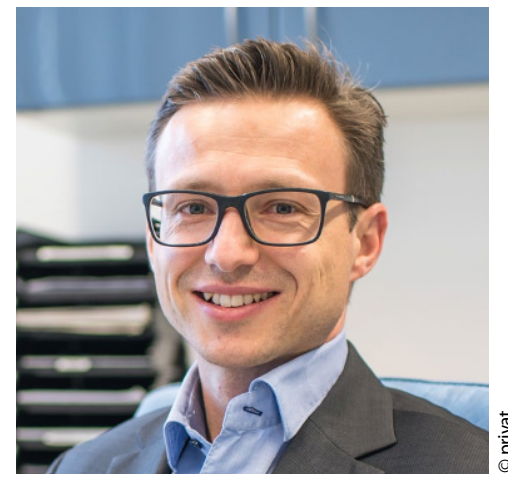

\ Prim. Prof. Dr. Christian Dejaco fentlich auch in weiteren Ländern umgesetzt wird.

Seit heuer gibt es die Summer School nicht nur für Studierende ...

Sautner: Die ÖGR hat inzwischen außerdem eine ÖGR Summer School für Jungärzte, sozusagen das Erweiterungs-Modul der Summer School für Studenten, ins Leben gerufen. Diese Summer School für Jungärzte ist eingebettet in ein mehrstufiges berufliches Entwicklungs- und Karrierekonzept, den ÖGR Karriere-Track.

Dejaco: Gegründet wurde die Summer School für Jung-Ärzte auf Initiative eines ehemaligen Grazer Absolventen der Summer School für Medizinstudenten, Philipp Bosch. Zielgruppe für die ÖGR Summer School für Jung-Ärzte sind junge Ärzte im Basisjahr, die vor der Entscheidung stehen, in welchem Bereich sie sich weiter entwickeln möchten. Ein weiteres Modul des ÖGR Karriere-Tracks ist ein Mentoring-Programm, in dessen Rahmen junge Rheumatologen vor allem in ihrer wissenschaftlichen Karriere unterstützt werden.

Sautner: Ein Student, der die Rheuma Summer School absolviert, kann in der Folge, während der Basisausbildung oder der internistischen Grundausbildung, an der Summer School für Jungärzte teilnehmen. Dort können über die ÖGR-Arbeitskreise Kontakte geknüpft werden,

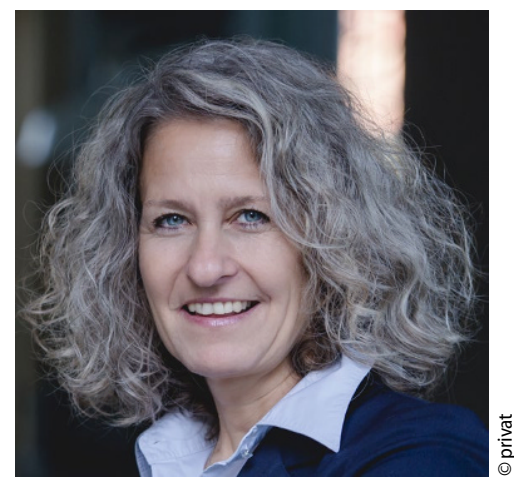

A Prim. Dr. Judith Sautner 
um in ein Mentoring-Programm aufgenommen zu werden.

\section{Welches Wissen wird in der Summer School für Medizinstudenten vermit- telt?}

Puchner: In den Vorträgen lernen die Studenten die Diagnose, Differentialdiagnose und Therapie rheumatischer Erkrankungen kennen. In den Workshops üben sie im Rahmen eines Bedside-Teachings: Wie untersucht man Rheuma-Patienten? Worauf ist zu achten? Wie macht man einen Gelenks-Ultraschall, wie eine Kapillarmikroskopie etc.?

Gibt es ein Nachwuchsproblem in der Rheumatologie?

Sautner: Die Rheumatologie ist im Ausbildungs-Curriculum kaum vertreten, es ist nicht möglich das Fach im Rahmen der Ausbildung umfassend zu präsentieren. Aber das ist eine Situation, die nicht nur unser Fach betrifft ...

Puchner: Ja, das erklärt auch, warum etwa die Gastroenterologen großes Interesse an unserer Summer School zeigen und ein ähnliches Projekt planen.

Sie möchten mit der Summer School auch Ihre Begeisterung für die Rheumatologie weitergeben. Was ist denn für Sie spannend an der Rheumatologie?

Dejaco: Für mich ist die Rheumatologie vielfältig, die Patientenfälle sind jeden Tag aufs Neue herausfordernd. Aber wir haben die Möglichkeit auch sehr kranken Patienten gut helfen zu können.

Puchner: Mich fasziniert an der Rheumatologie, dass sie die gesamte innere Medizin umfasst. Zu $70 \%$ erfolgen die Diagnosen durch Anamnese und Untersuchung. Natürlich brauchen wir auch Geräte, aber wir arbeiten in engem Kontakt mit den Patienten und mit Kollegen anderer Fachdisziplinen.

\section{Der ÖGR Karriere-Track}

\section{Die ÖGR/EULAR Summer School für Studierende}

Im Rahmen der Summer School können drei Tage lang Vorträge und Kleingruppen-Workshops mit internationalen Rheumatologen besucht werden.

Wer an der 6. ÖGR/EULAR Summer School teilnehmen möchte, kann eine Bewerbung samt Motivationsschreiben an das Sekretariat der ÖGR senden: office@rheumatologie.at

Die Summer School wird als freies Wahlfach von den Universitäten angerechnet. Der Kurs richtet sich in erster Linie an Studierende der fünf österreichischen Medizinuniversitäten im 5. und 6. Studienjahr.

\section{Die ÖGR Summer School für Jungärzte}

Die ÖGR Summer School für Jungärzte richtet sich in erster Linie an junge Ärztinnen und Ärzte in klinischer und/ oder wissenschaftlicher Ausbildung für das Fach Innere Medizin und Rheumatologie, sowie auch an Jungärzte mit dem Ziel Rheumatologen zu werden.

Der Fokus dieser Veranstaltung liegt auf Career Development, Networking und Mentoring in der österreichischen Rheumatologie-Landschaft.

\section{Das ÖGR Mentoring-Programm}

Die dritte Stufe des ÖGR Karriere-Tracks ist das Mentoring-Programm. Hier werden junge Rheumatologen in ihrer klinischen und wissenschaftlichen Karriere unterstützt.

Weitere Informationen finden Sie auf der Website der Österreichischen Gesellschaft für Rheumatologie und Rehabilitation: www.rheumatologie.at

Sautner: Die Rheumatologie ist ein vielfältiges, medizinisch und menschlich forderndes, aber auch sehr bereicherndes Fach. Wer gerne mit Menschen redet, sie gerne klinisch untersucht und ganzheitlich erfasst und betreut, der ist in der Rheumatologie gut aufgehoben.

Unsere Begeisterung für die Rheumatologie ist offenbar ansteckend, denn wenn wir in der Summer School über unser Fach sprechen, sind auch die Studenten begeistert. Sie merken, dass wir auch miteinander viel reden, denn die Rheumatologie lebt stark von der Interaktion, vom Gespräch mit den Patienten, mit den Kollegen und vom interdisziplinären Austausch. Das und die gute Stimmung, die bei der Summer School herrscht, fällt den Studenten besonders oft positiv auf.

Was ist die Zukunft der Summer School?

Puchner: Wir möchten die ÖGR Summer School auf jeden Fall weiterführen und weiter entwickeln. Wir lassen jede Summer School von den Teilnehmern evaluieren um die Summer School weiter zu optimieren.

Dejaco: Wenn andere Länder das Modell der Summer School übernehmen, wäre das natürlich ein großer Erfolg. Ansonsten hoffen wir, dass weiterhin viele Studenten von unseren drei Ausbildungsmodulen profitieren.

Sautner: Nachwuchsförderung ist die beste Investition in die Zukunft der Rheumatologie.

Das Gespräch führte

Mag. Tanja Fabsits

Hinweis des Verlags. Der Verlag bleibt in Hinblick auf geografische Zuordnungen und Gebietsbezeichnungen in veröffentlichten Karten und Institutsadressen neutral.

rheuma plus $2021 \cdot 20: 232-235$

https://doi.org/10.1007/s12688-

021-00483-2

(c) Springer-Verlag GmbH Austria, ein Teil

von Springer Nature 2021 